\title{
Felicidade e satisfação com a vida: voz de mulheres adultas e idosas
}

\author{
Marilene Rodrigues Portella, ${ }^{1}$ Helenice de Moura Scortegagna, ${ }^{2}$ Nadir Antônio Pichler,${ }^{3}$ Daniela Bertol Graeff ${ }^{4}$
}

\section{Resumo}

Envelhecer bem está intimamente ligado à satisfação com a vida e a felicidade, considerando-se a capacidade cognitiva do sujeito, os elementos constituintes da experiência subjetiva e o alcance dos objetivos individuais. A partir dessa compreensão e com objetivo de conhecer e descrever as fontes de felicidade e de satisfação com a vida na concepção de adultos e idosos, foi desenvolvido um estudo qualitativo, ancorado no método do Grupo Focal (GF). Participaram 22 mulheres com idade entre 49 e 81 anos, matriculadas em um programa de extensão na modalidade de Universidade Aberta para a terceira idade, vinculada a uma instituição de ensino superior, localizada em um município no sul do Brasil. Os dados foram coletados em 2014, provenientes de três encontros, com duração média de 60 minutos cada um. O estudo revelou que a maioria das mulheres está satisfeita com a vida e sente-se feliz, ape- sar das adversidades enfrentadas ao longo do processo de viver e envelhecer. As muIheres consideram a família, a presença de Deus, a saúde e a paz como principais fontes de satisfação com a vida e os principais elementos geradores da felicidade. Esses dados evidenciam a velhice como um momento que pode ser vivido e experienciado de forma prazerosa, considerando a multidimensionalidade e a integralidade do ser. Ressalta-se a importância da compreensão de tais indicadores nas ações implementadas pelos profissionais atuantes no campo da gerontologia, com vistas à qualificação do viver e do envelhecer do ser humano, pois não basta almejar uma vida longa, mas, sim, a qualidade para viver sua extensão.

Palavras-chave: Envelhecimento. Felicidade. Gerontologia. Planejamento em Saúde e Bem-Estar. Saúde da Mulher.

1 Enfermeira. Doutora em Enfermagem pela Universidade Federal de Santa Catarina. Docente Titular do Instituto de Ciências Biológicas e do Programa de Pós-Graduação em Envelhecimento Humano (PPGEH) da Universidade de Passo Fundo. E-mail: portella@upf.br

2 Enfermeira. Doutora em Enfermagem pela Universidade Federal de Santa Catarina. Docente Titular do Instituto de Ciências Biológicas e do Programa de Pós-Graduação em Envelhecimento Humano (PPGEH) da Universidade de Passo Fundo. E-mail: helenice@upf.br

3 Doutor em Filosofia pela Pontifícia Universidade Católica do Rio Grande do Sul (PUCRS). Docente do Programa de Pós-Graduação em Envelhecimento Humano (PPGEH) da Universidade de Passo Fundo. E-mail: nadirp@upf.br

4 Fisioterapeuta. Mestre em Epidemiologia pela Universidade Federal do Rio Grande do Sul (Ufrgs). Coordenadora do Centro de Referência e Atenção ao Idoso da Universidade de Passo Fundo (Creati/UPF). Docente da Faculdade de Medicina da Universidade de Passo Fundo (UPF). E-mail: danibertol@upf.br

$\rightarrow$ http://dx.doi.org/10.5335/rbceh.v13i2.5960

Recebido em: 19.04.2016. Aceito em: 04.08.2017. 


\section{Introdução}

O sentimento de felicidade tem sido considerado como um dos melhores indicadores de bem-estar. Desse modo, esse fator atua positivamente, propiciando melhoria nas condições de vida e de saúde dos idosos (LIMA; BARROS; ALVES, 2012).

Assim, a busca pela felicidade, o bem supremo na vida de uma pessoa, sempre foi algo almejado no decorrer da história da humanidade. Nas éticas antigas, por exemplo, era um tema recorrente, palco de muitas pesquisas e discussões. Atualmente, devido aos progressos econômicos, políticos, culturais e tecnológicos da humanidade, a felicidade novamente está em voga, naturalmente estruturada em outros valores. Para muitos, a felicidade restringe-se aos atos de trabalhar, produzir, ganhar dinheiro, consumir, seguir a moda, satisfazer desejos de forma compulsiva e imediata, etc. Porém, a busca por uma felicidade mais consistente segue referências mais profundas, como a realização pessoal, execução de um projeto de vida com propósitos, enaltecendo qualidades subjetivas ou da alma, como o cuidado de si, a autoestima, o autoconhecimento, a satisfação racional dos desejos e prazeres, a participação em grupos de convivência, o convívio familiar, a saúde integral, a autonomia, a dignidade, a sexualidade, a espiritualidade, a paz, etc. (LUZ; AMATUZZI, 2008; WIBELINGER, 2014; PESSINI, 2014).

Satisfação é um estado subjetivo do ser humano. É um julgamento cognitivo de difícil mensuração, todavia reflete, em parte, o bem-estar subjetivo individual, considerando os motivos que levam as pessoas a viverem suas experiências de vida e o modo como o fazem (JOIA; RUIZ; DONALISIO, 2007). O envelhecer bem pode estar intimamente ligado à satisfação com a vida e a felicidade, nas quais se encontram elementos da experiência subjetiva em que se valoriza o otimismo, a esperança, a religiosidade, o trabalho, a família, as amizades, a saúde física (DENDENA et al., 2011), bem como o alcance dos objetivos individuais e a capacidade cognitiva do sujeito.

O estudo do bem-estar subjetivo (BES) tem como escopo central compreender a avaliação que as pessoas fazem de suas vidas, podendo receber outras denominações, tais como felicidade, satisfação, estado de espírito, afeto positivo, sendo também considerado, por alguns autores, como avaliação subjetiva da qualidade de vida. Por isso, há uma tendência contemporânea para relacionar a felicidade à promoção de saúde (SCORSOLINI-COMIN, 2010).

O estudo de Luz e Amatuzzi (2008) revela que a experiência de felicidade para idosos incide sobre aqueles que se adaptam melhor às transformações trazidas pelo envelhecimento, nas pessoas que conseguem manter um equilíbrio entre as perdas e os ganhos. Assim, a vivência desse sentimento nos idosos está relacionada ao bem-estar de sua família, ao fato de poderem trabalhar, à expressão da fé, às relações sociais e à amizade. 
Considerando esse contexto, o objetivo do estudo foi conhecer e descrever as fontes de felicidade e satisfação com a vida na concepção de adultos e idosos.

\section{Metodologia}

Trata-se de um estudo exploratório, descritivo, de abordagem qualitativa.

A pesquisa qualitativa procura investigar, identificar e descrever informações de realidades subjetivas de pessoas e grupos sociais, ou seja, suas crenças, opiniões, hábitos, valores, atitudes, aspirações, ideologias, com a finalidade de reuni-las e sintetizá-las para compreender suas razões históricas, culturais e atuais (MINAYO, 2013).

Optou-se pelo método do grupo focal (GF) por entender que essa técnica busca capturar formas de linguagem, significações, expressões e tipos de comentários de determinado segmento, além de alcançar níveis crescentes de compreensão e aprofundamento de um tema, a partir de debates focados em assuntos específicos (GATTI, 2005). O grupo focal permite "a compreensão de práticas cotidianas, atitudes e comportamentos prevalecentes no trabalho com alguns indivíduos que compartilham traços em comum" (LOPES, 2014, p. 484).

Para compor os GFs, inicialmente, foi apresentado o projeto para o Coordenador de um programa de extensão nos moldes de uma universidade aberta para a terceira idade, vinculados a uma universidade; posteriormente, foi estendido o convite às pessoas, ocasião em que foi apresentada a proposta de pesquisa com vistas ao recrutamento das participantes. Compuseram a amostra 22 mulheres com idades entre 49 e 81 anos, casadas, com diferentes níveis de escolaridade e de condições socioeconômica, residentes numa cidade do interior do norte do estado do Rio Grande do Sul. As participantes foram distribuídas em três grupos. O GF1 foi composto por dez mulheres com idades entre 52 e 81 anos; no GF2, participaram dez mulheres com idades entre 53 e 74 anos. O GF3 atuou como o grupo de validação interna e, na sua composição, contou com oito mulheres, com idades entre 49 e 81 anos. $\mathrm{O}$ cenário e os horários das sessões dos GFs foram determinados em comum acordo com os participantes e com a anuência da coordenação. As sessões dos GFs tiveram como moderador e observador pesquisadores da equipe. Foram realizados três encontros, um por grupo, com duração média de 60 minutos cada um. O período de coleta de dados ocorreu em 2014. As sessões dos GF1 e GF2 começaram com a apresentação das participantes, na sequência, depois de esclarecida a dinâmica do encontro, foram lançadas as seguintes perguntas: $\mathrm{O}$ que traz satisfação na vida? O que traz felicidade? $\mathrm{Na}$ sessão do GF3, com a iniciativa de validar os achados nos grupos anteriores, como elemento desencadeador no debate no grupo, foram apresentadas as sínteses obtidas nos GF1 e GF2. Para a análise e a interpretação das informações, foi considerada a perspectiva de análise dos GFs proposta por Gatti (2005). Reuniram-se as anotações do observador, as sínteses apreendidas nas sessões e as transcrições dos encontros. Com a posse desse material, deu-se 
início ao processo de leitura e releitura para fins de codificação. A frequência das menções nessas unidades orientou o roteiro para a interpretação dos dados. A análise aconteceu numa dimensão interacionista, e a interpretação, ancorada no referencial construído. O projeto foi aprovado pelo Comitê de Ética em Pesquisa da Universidade de Passo Fundo (Protocolo n. 393/2011).

\section{Resultado e discussão}

A satisfação na vida e a felicidade podem ser expressas por condições ou valores manifestados em diversas formas. Neste estudo, quando solicitado para as participantes que se manifestassem sobre a temática, afloraram variadas expressões, sintetizadas e apresentadas na Figura 1.

Figura 1 - Felicidade e satisfação com a vida evocada em palavras



Fonte: figura elaborada pelos pesquisadores.
A maioria das mulheres se diz satisfeita com a vida e se sente feliz, mesmo com as adversidades enfrentadas no decorrer de suas existências. Os resultados do presente trabalho revelaram que as participantes, independentemente do estado conjugal, consideram a família a maior fonte de satisfação com a vida e o elemento fundamental gerador da felicidade.

No debate dos GFs, expressões como "estar bem com a família", "o amor entre as pessoas da família", "presença dos netos", "o nascimento do filho", "a família estar feliz", "o amor na família", "ver a família feliz", "família toda unida", "a família é a base de tudo" ilustram o valor que a família assume na vivência das mulheres.

A família tem sido apontada como sendo a fonte prioritária de suporte e apoio à pessoa no curso da vida, em especial na velhice (RESENDE et al., 2007).

Um estudo desenvolvido por Molly (2017), com quatro adultos maiores, nos Estados Unidos da América, conclui que a satisfação com a vida, a autoestima e a felicidade são decorrentes do convívio com familiares e amigos, diminuindo, inclusive, os índices de depressão.

Algumas das pessoas manifestaram ser felizes por estarem contentes com a presença ou a visita frequente dos netos, assim como por observarem o sucesso alcançado pela família, ilustrada pela expressão "ver a evolução da vida dos filhos e dos netos, isso dá satisfação e traz felicidade".

De acordo com um estudo realizado sobre vivências de felicidade de pessoas 
idosas, Luz e Amatuzzi (2008) constataram que a dimensão familiar é a fonte e a provedora de felicidade, portadora de estabilidade e proteção, porque é a estrutura fundamental em que o ser humano está inserido numa sociedade. Ainda, "a família deve ajudar o idoso a viver melhor, não como um peso, mas como integrante do sistema familiar" (2008, p. 306).

Nota-se nas palavras evocadas que a saúde está entre os aspectos que promovem satisfação com a vida e a felicidade, independentemente do contexto em que as pessoas vivem (DOLL, 1999), bem como saúde, ambiente familiar e independência estão entre os principais determinantes da felicidade como apontam estudos de longa data, "como determinantes de boa qualidade de vida" (JOIA; RUIZ; DONALISIO, 2007, p. 135). Da mesma forma, Moraes e Souza (2005) associam satisfação com a vida ao relacionamento com os familiares e amigos, à saúde e ao bem-estar.

Nesse alinhamento, Ribeiro (2010) ressalta que a qualidade de vida entre os mais velhos está associada ao poder contar com a família e ao prazer de ter uma boa saúde, o que foi reforçado pelas mulheres ao se manifestarem: "O que traz satisfação na vida é o amor, são os filhos, a união da família e ter saúde", "Saúde, paz e o amor deixam as pessoas felizes", "ter saúde é uma riqueza, é uma graça de Deus, isso é felicidade".

A saúde é decorrente de muitos fatores, sendo um dos alicerces da velhice bem-sucedida, fundada em estilos de vida que mantêm o corpo e a mente saudáveis por meio do exercício e da atividade física, de bons hábitos de nutrição e envolvimento em atividades interessantes que desafiam a mente. $\mathrm{O}$ desenvolvimento desses hábitos relaciona-se com a educação e as atitudes da família e de amigos que valorizam a vida saudável e produtiva (NOBRE et al., 2013; LAWLER; YOUNGER, 2002; MOLLY, 2017).

As vivências de felicidade das participantes estão relacionadas ao amor a Deus e ao fato de sentirem que existe amor entre as pessoas. Para Dendena et al. (2011), a religiosidade é vista como uma das forma de assegurar que o envelhecimento não seja caracterizado como etapa de abandono e solidão. Para os autores, há uma tendência de a religiosidade aumentar à medida que as pessoas envelhecem, em função da proximidade da morte, fazendo com que os idosos se aproximem e exercitem mais suas crenças no sagrado, pois a iminência da finitude pode estender o desejo pela oração em busca de conforto espiritual. Maldaun et al. (2008) ressaltam que a inclusão da religiosidade na vivência dos idosos assume papel fundamental, pois atribui significado à vida das pessoas, influenciando no seu bem-estar subjetivo.

A pesquisa desenvolvida por Chaves et al. (2014, p. 651), com 287 idosos em uma unidade da Estratégia Saúde da Família, sobre qualidade de vida, depressão e o significado da religiosidade, concluiu que " $97 \%$ consideram a religião como importante ou muito importante em suas vidas". A religião é geradora de suporte psicológico, propiciadora de 
satisfação com a vida, felicidade, autoestima e moral elevada.

Para Cocentino e Viana (2011), a finitude existencial traz inúmeras preocupações psicológicas, como o medo da própria morte e do processo de morrer e a perda de pessoas queridas, capaz de gerar ansiedade, angústia e impotência.

Entretanto, a fé, a esperança e a confiança em Deus são crenças e motivações fundamentais na vida de pessoas (GALL; MALETTE; GUIRGUIS-YOUNGER, 2011). Um estudo realizado por Gutz e Camargo (2013), com 30 idosos, apresenta a espiritualidade como um dos recursos para enfrentar "situações adversas, constituindo-se de aspectos emocionais e motivacionais na busca de um significado para a vida" (2013, p. 793). A espiritualidade, ainda, é geradora de felicidade geral, de saúde física, psíquica e mental, pois favorece a resiliência, o envelhecimento bem-sucedido, a força e a proteção divina para enfrentar os desafios da vida cotidiana. Espiritualidade é a capacidade de transcendência, um meio de conexão com a divindade, portadora de fé e sentido para a vida (LAWLER; YOUNGER, 2002; POWELL; SHAHABI; THORESEN, 2003; SOLOMON, 2003).

As mulheres deste estudo se manifestaram acerca do respeito entre as pessoas, o companheirismo, a amizade e o lazer associado à felicidade e à satisfação com a vida. Estudos pronunciam que as mulheres têm uma maior preocupação com o lazer, assim como com as relações interpessoais (YOKOYAMA; CARVALHO; VIZZOTTO, 2006; MOLLY, 2017).
As relações interpessoais, por exemplo entre jovens e idosos, reforçam a importância da educação intergeracional, porque essa forma de convivência gera satisfação com a vida e felicidade, fazendo com que os mais jovens aprendam "a enxergar os mais velhos como pessoas ativas, produtivas e participativas, e os mais velhos podem ensinar muito de suas experiências e valores morais, como o respeito, para os mais jovens" (MAIO; LOLLI, 2015, p. 408).

\section{Considerações finais}

Pode-se evidenciar que os vínculos e as relações familiares, bem como a saúde e a espiritualidade, são dimensões fundamentais para a felicidade e o bem-estar dessas mulheres. Esses dados revelam a velhice como um momento que pode ser vivido e experienciado de forma prazerosa, considerando a multidimensionalidade e a integralidade do ser.

$O$ estudo demonstrou que a satisfação com a vida foi relacionada à proximidade com a família. Para essas mulheres, de diferentes idades, a felicidade e a satisfação com a vida estão na presença da família, no amor, na paz e na união entre os seus, pois são os elementos responsáveis pela promoção do bem. A presença de Deus e a saúde são fundamentais, associadas às experiências de amizade, companheirismo e lazer, sendo para essas mulheres os indicadores de felicidade e satisfação com a vida.

A partir do evidenciado neste estudo, somado aos demais estudos na temática, espera-se contribuir para que 
os profissionais que atuam no campo da gerontologia compreendam melhor os indicadores que qualificam o viver e o envelhecer do ser humano, em especial, das mulheres. Nesse sentido, buscar medidas que propiciem um envelhecimento com qualidade deve ser a meta de todos aqueles que se preocupam com o cuidado de si e do outro, pois não basta almejar uma vida longa, mas, sim, a qualidade para viver sua extensão.

Considerando os fatores físicos, a natureza genética, o estilo de vida, entre outros, a felicidade na longevidade é um processo complexo, um produto intrinsecamente ligado à forma de vida, pois envolve autoestima, autoconhecimento, cuidado de si, autonomia, independência financeira, saúde, participação, segurança (OMS, 2005).

Ainda, a satisfação com a vida, na longevidade, pode estar associada com a prática das virtudes da amizade, da cordialidade, da hospitalidade, da confiança, da espiritualidade, da compaixão, da prudência, do equilíbrio, do autocontrole, tudo isso acompanhado pelo convívio social e familiar. Como vimos neste estudo, vida ativa, vida meditativa, vivências da sexualidade e do erotismo, alimentação saudável, atividade física, memória ativa, entre outros fatores, são geradores de envelhecimento saudável, definido como um "processo de desenvolvimento e manutenção da capacidade funcional que permite o bem-estar em idade avançada" (OMS, 2005, p. 13).

\section{Happiness and satisfaction with life: voices of adults and elderly women}

\section{Abstract}

Ageing well may be closely linked to life satisfaction and happiness, considering the cognitive ability of the subject, the elements of subjective experience and the achievement of individual goals. From this understanding, and in order to discover and describe the sources of happiness and life satisfaction from the view of adults and elderly was developed a qualitative study, anchored in the method of the Focus Group. Participants were 22 women aged 49 to 81 years, enrolled in an outreach program in the form of Open University for the Third Age linked to a higher education institution, located in a city in southern Brazil. Data were collected in 2014, from three meetings, with an average duration of 60 minutes each. The study revealed that most women are satisfied with life and feel happy despite the adversities they face throughout the process of living and aging. Women consider family, the presence of God, health and peace as the main source of life satisfaction and the main happiness generating element. These data reveal the old age as a time that can be lived and experienced in a pleasant way, considering the multidimensionality and completeness of being. It emphasizes the importance of understanding these indicators of the actions implemented by the professional's actions in the field of gerontology, with a view to qualifying the living and the aging of the humans, it does not just aim for a long life, but the quality to live its length.

Keywords: Aging. Happiness. Health and Wellness Planning. Gerontology. Women's Health. 


\section{Referências}

CHAVES, E. C. L. et al. Qualidade de vida, sintomas depressivos e religiosidade em idosos: um estudo transversal. Texto Contexto Enferm, Florianópolis (SC), v. 23, n. 3, p. 648-655, jul./set. 2014. Disponível em: <http://www.scielo.br/pdf/tce/v23n3/pt_01040707-tce-23-03-00648.pdf>. Acesso em: $1^{\text {o }}$ mar. 2017.

DENDENA, A. et al. Religiosidade e envelhecimento bem-sucedido. Unoesc \& Ciência - ACHS, Joaçaba (SC), v. 2, n. 2, p. 184-196, jul./dez. 2011.

COCENTINO, J. M. B.; VIANA, T. C. A velhice e a morte: reflexões sobre o processo de luto. Revista Brasileira de Geriatria e Gerontologia, Rio de Janeiro (RJ), v. 14, n. 3, p. 591-599, ago./set. 2011.

DOLL, J. Satisfação de vida de homens e mulheres idosos no Brasil e na Alemanha. Cadernos Pagu, (Unicamp. Impresso), Campinas (SP), v.13, p. 109-159, 1999.

JOIA, L. C.; RUIZ, T.; DONALISIO, M. R. Condições associadas ao grau de satisfação com a vida entre a população de idosos. Rev. Saúde Pública, São Paulo (SP), v. 41, n. 1, p.131-138, fev. 2007. Disponível em: <http:// www.scielo.br/pdf/rsp/v41n1/19.pdf >. Acesso em: $1^{\circ}$ abr. 2017.

GALL, T. L.; MALETTE, J.; GUIRGUISYOUNGER, M. Spirituality and religiousness: a diversity of definitions. $J$ Spiritual Mental Health, Toronto (Ontariov), v. 13, n. 3, p. 158-181, 2011.

GUTZ, L.; CAMARGO, B. V. Espiritualidade entre idosos mais velhos: um estudo de representações. Rev. Bras. Geriatr. Gerontol., Rio de Janeiro (RJ), v. 16, n. 4, p. 793-804, out./dez. 2013.

GATTI, B. A. Grupo focal na pesquisa em Ciências sociais e humanas. Brasília: Líber Livro, 2005.
LAWLER, K. A.; YOUNGER, J. W. Theobiology: an analysis of spirituality, cardiovascular responses, stress, mood, and physical health. Journal of Religion and Health New York, NY, v. 41, n. 4, p. 347-62, 2002.

LIMA, M. G.; BARROS, M. B. A.; ALVES, M. C. G. P. Sentimento de felicidade em idosos: uma abordagem epidemiológica, ISA-Camp 2008. Cad. Saúde Pública, São Paulo (SP), v. 28 , n. 2 , p. 2280-2292, dez. 2012.

LOPES, B. E. M. Grupo focal na pesquisa em ciências sociais e humanas. Revista Educação e Políticas em Debate, Uberlândia (GO), v. 3, n. 2, p. 482-492, ago./dez. 2014.

LUZ, M. M. C.; AMATUZZI, M. M. Vivências de felicidade de pessoas idosas. Estudos de Psicologia, Campinas (SP), v. 25, n. 2, p. 303307, abr.jun. 2008.

MALDAUN, D. et al. Espiritualidade / Religiosidade. In: NERI, A. L. (Org.). Palavras-chave em Gerontologia. Campinas: Alínea, 2008. p. 70-73.

MINAYO, M. C. S. O desafio do conhecimento: pesquisa qualitativa em saúde. 13. ed. São Paulo: Hucitec, 2013.

MOLLY, C. Effects of Life Review on $H$ appiness and Life Satisfaction in Older Adults. 2015. Disponível em: <http://digitalcommons. wku.edu/cgi/viewcontent.cgi? article $=2525$ \&context=theses $>$. Acesso em: $1^{\circ}$ abr. 2017.

MAIO, E. R.; LOLLI, M. C. S. Educação permanente, não formal, como instrumento para a valorização e o reconhecimento do idoso: o modelo da Unati/UEM. Ensino Em Re-Vista, Uberlândia (GO), v. 22, n. 2, p.401-410, jul./ dez. 2015.

MORAES, J. F. D.; SOUZA, V. B. A. Factors associated with the successful aging of the socially-active elderly in the metropolitan region of Porto Alegre. Revista Brasileira de Psiquiatria, São Paulo (SP), v. 27, n. 4, p. 302-308, dez. 2005. 
NOBRE, S. et al. Felicidade: Amor e Arte. International Journal of Developmental and Educational Psychology. Revista de Psicología, Fortaleza (CE), v. 2, n. 1, p. 421-430, jan./jun. 2013.

PESSINI, L. Bioética, espiritualidade e a arte de cuidar em saúde. In: SIVIERO, I.; ROSIN, N. (Org.). Envelhecimento humano, espiritualidade e cuidado: vol. II: Espiritualidade e missão. Passo Fundo: IFIBE, 2014. p. 31-83.

POWELL, L. H.; SHAHABI, L.; THORESEN, C. E. Religion and spirituality. Linkages to Physical Health. American Psychologist., Washington, v. 58, n. 1, p. 36-52, 2003.

RESENDE, M. C. et al. Rede de relações e satisfação com a vida em pessoas com amputação de membros. Ciências \& Cognição, Rio de Janeiro (RJ), v. 10, p. 164-177, jan./ jun. 2007.

RIBEIRO, L. F. Qualidade de vida na terceira idade. Ágora: R. Divulg. Cient., Mafra (SC) v. 17 , n. 2 , p. $75-80,2010$.

SCORSOLINI-COMIN, F.; SANTOS, M. A. dos. O estudo científico da felicidade e a promoção da saúde: revisão integrativa da literatura. Rev. Latino-Am. Enfermagem, Ribeirão Preto (SP), v. 18, n. 3, p. 189-199, maio/jun. 2010.

SOLOMON, R. C. Espiritualidade para céticos: paixão, verdade cósmica e racionalidade no século XXI. Tradução de Maria Luíza X. de A. Borges. Rio de Janeiro: Civilização Brasileira, 2003.

YOKOYAMA, C. E.; CARVALHO, R. S.; VIZZOTTO, M. M. Qualidade de vida na velhice segundo a percepção de idosos frequentadores de um centro de referência. Psicólogo inFormação, São Paulo (SP), v. 10, n. 10, p. 57-82, jan./dez. 2006.

WIBELINGER, L. M. Segredos para envelhecer bem. Passo Fundo: IFIBE, 2014.
ORGANIZAÇÃO MUNDIAL DA SAÚDE (OMS). Envelhecimento ativo: uma política de saúde. Brasília, DF: Organização Pan-Americana da Saúde (OPAS), 2005. 